\title{
Changes in Near-Infrared Spectroscopy After Congenital Cyanotic Heart Surgery
}

\begin{abstract}
Judith Ju-Ming Wong ${ }^{1,2 *}$, Ching Kit Chen ${ }^{3}$, Rajesh Babu Moorakonda ${ }^{4,5}$, Olivia Wijeweera ${ }^{6}$, Tracy Yi Shuen Tan ${ }^{6}$, Masakazu Nakao ${ }^{7}$, John Carson Allen Jr., Tsee Foong Loh ${ }^{1,2}$ and Jan Hau Lee ${ }^{1,2}$

'Children's Intensive Care Unit, Department of Paediatric Subspecialties, KK Women's and Children's Hospital, Singapore, Singapore, ${ }^{2}$ Duke-NUS Medical School, Singapore, Singapore, ${ }^{3}$ Cardiology Services, Department of Paediatric Subspecialties, KK Women's and Children's Hospital, Singapore, Singapore, ${ }^{4}$ Singapore Clinical Research Institute, Singapore, Singapore, ${ }^{5}$ Duke-NUS Medical School, Centre for Quantitative Medicine, Singapore, Singapore, ${ }^{6}$ Department of Paediatric Anaesthesia, KK Women's and Children's Hospital, Singapore, Singapore, ${ }^{7}$ Department of Paediatric Cardiothoracic Surgery, KK Women's and Children's Hospital, Singapore, Singapore
\end{abstract}

Background: Since oxygen saturation from pulse oximetry $\left(\mathrm{SpO}_{2}\right)$ and partial pressure of arterial oxygen $\left(\mathrm{PaO}_{2}\right)$ are observed to improve immediately after surgical correction of cyanotic congenital heart disease $(\mathrm{CHD})$, we postulate that cerebral $\left(\mathrm{CrO}_{2}\right)$ and somatic $\left(\mathrm{SrO}_{2}\right)$ oximetry also improves immediately post-correction. We aim to prospectively examine $\mathrm{CrO}_{2}$ and $\mathrm{SrO}_{2}$, before, during, and after surgical correction as well as on hospital discharge in children with cyanotic CHD to determine if and when these variables increase.

Methods: This is a prospective observational trial. Eligibility criteria included children below 18 years of age with cyanotic CHD who required any cardiac surgical procedure. $\mathrm{CrO}_{2}$ and $\mathrm{SrO}_{2}$ measurements were summarized at six time-points for comparison: (1) pre-cardiopulmonary bypass (CPB); (2) during CPB; (3) post-CPB; (4) Day 1 in the pediatric intensive care unit (PICU); (5) Day 2 PICU; and (6) discharge. Categorical and continuous variables are presented as counts (percentages) and median (interquartile range), respectively.

Results: Twenty-one patients were analyzed. 15 (71.4\%) and 6 (28.6\%) patients underwent corrective and palliative surgeries, respectively. In the corrective surgery group, $\mathrm{SpO}_{2}$ increased immediately post-CPB compared to pre-CPB $[99(98,100)$ vs. 86\% $(79,90)$; $p<0.001]$ and remained in the normal range through to hospital discharge. Post-CPB $\mathrm{CrO}_{2}$ did not change from pre-CPB $[72.8(58.8,79.0)$ vs. $72.1 \%(63.0,78.3) ; p=0.761]$ and even decreased on hospital discharge $[60.5$ (53.6, 62.9) vs. $72.1 \%(63.0,78.3)$; $p=0.005]$. Post-CPB $\mathrm{SrO}_{2}$ increased compared to pre-CPB $[87.3(77.2,89.5)$ vs. $72.7 \%$ (65.6, 77.3); $p=0.001$ ] but progressively decreased during PICU stay to a value lower than baseline at hospital discharge $[66.9(57.3,76.9)$ vs. $72.7 \%(65.6,77.3) ; p=0.048]$.

Conclusion: $\mathrm{CrO}_{2}$ and $\mathrm{SrO}_{2}$ did not increase after corrective surgery of cyanotic $\mathrm{CHD}$ even up to hospital discharge. Future larger studies are required to validate these findings. (This study is registered with ClinicalTrials.gov ID: NCT02417259.)

Keywords: congenital heart disease, congenital heart surgery, children, near-infrared spectroscopy, perioperative care, pediatric, regional oximetry 


\section{INTRODUCTION}

Adoption of near-infrared spectroscopy (NIRS) is increasing in pediatric cardiac anesthesia and pediatric intensive care units (PICUs) across North America and Europe (1). The two most common sites for regional NIRS are cerebral $\left(\mathrm{CrO}_{2}\right)$ and somatic $\left(\mathrm{SrO}_{2}\right)$ oximetry. Proposed utility of NIRS include: (1) pre-operative risk stratification; (2) monitoring for uncommon events; and (3) guide physiologic interventions (2). However, significant debate remains on the interpretation of NIRS monitoring because of the lack of universally accepted normal/abnormal values, definition of a threshold event and standardized management algorithm $(1,3)$.

Despite current limitations, cerebral oximetry has been validated with other regional oxygenation measures (e.g., jugular venous bulb saturations, superior vena cava saturations) and global oxygenation measures (e.g., serum lactate, mixed venous saturations) (4-6). Associations between low NIRS and poor long-term neurological outcomes (e.g., neurodevelopmental scales, magnetic resonance imaging changes) have been reported $(7,8)$. Conversely, however, there are also studies reporting failure of NIRS to correlate with oxygenation measures or clinical outcomes (9). Moreover, the natural history of tissue oximetry over a longitudinal perioperative period in children with congenital heart disease (CHD), especially those with cyanotic CHD undergoing cardiac surgery is not well studied. Early studies reported conflicting data as to whether baseline $\mathrm{CrO}_{2}$ was decreased in patients with cyanotic and acyanotic CHD $(10,11)$. There is sparse longitudinal data across the perioperative period (12).

Since oxygen saturations from pulse oximetry $\left(\mathrm{SpO}_{2}\right)$ and partial pressure of arterial oxygen $\left(\mathrm{PaO}_{2}\right)$ are observed to improve immediately after surgical correction of cyanotic CHD, we postulate that $\mathrm{CrO}_{2}$ and somatic $\left(\mathrm{SrO}_{2}\right)$ oximetry would also improve immediately post-correction. Therefore, we undertook this pilot study to examine $\mathrm{CrO}_{2}$ and $\mathrm{SrO}_{2}$, before, during and after surgical correction as well as on hospital discharge in these children to determine if and when these variables increase.

\section{MATERIALS AND METHODS}

\section{Patients}

This prospective observational study was approved by the Centralized Institutional Review Board of our hospital. Both parental informed and written consent was obtained and patients were enrolled between July 2015 and June 2016 at KK Women's and Children's Hospital, Singapore. Eligibility criteria included patients below 18 years of age with cyanotic CHD who required any cardiac surgical procedure. These surgeries can be either full corrective or staged procedures. Exclusion criteria included

\footnotetext{
Abbreviations: $\mathrm{CaO}_{2}$, arterial oxygen content; $\mathrm{CBF}$, cerebral blood flow; $\mathrm{cFOE}$, cerebral fractional oxygen extraction; CHD, congenital heart disease; $\mathrm{CPB}$, cardiopulmonary bypass; $\mathrm{CRMO}_{2}$, cerebral metabolic rate of oxygen; $\mathrm{CrO}_{2}$, cerebral oximetry; DHCA, deep hypothermic circulatory arrest; NIRS, near-infrared spectroscopy; $\mathrm{PaO}_{2}$, partial pressure of arterial oxygen; $\mathrm{PICU}$, pediatric intensive care unit; $\mathrm{SpO}_{2}$, pulse oximetry; $\mathrm{SrO}_{2}$, somatic oximetry; $\mathrm{SvO}_{2}$, cerebral venous oxygenation.
}

premature infants of less than a corrected age of 35 weeks and congenital lactic acidosis syndromes.

\section{Anesthesia and Perfusion Methods}

The anesthesia induction technique was at the discretion of the attending anesthetist. Inhaled anesthetic used was sevoflurane. Intravenous agents included morphine, fentanyl, ketamine, midazolam, propofol, rocuronium, and dexmedetomidine. After induction of anesthesia, standard monitoring included: central venous catheter in the right or left internal jugular vein for central venous pressure monitoring; radial or femoral artery catheter for measurement of systemic arterial blood pressure; indwelling urinary catheter for monitoring urinary output and; esophageal/nasopharyngeal and rectal temperature probes to measure core body temperature. Core cooling was achieved through the cardiopulmonary bypass (CPB) circuit. The bypass circuit was primed with packed cells. Pump flow rates were maintained at $100-150 \mathrm{ml} / \mathrm{kg} / \mathrm{min}$ to achieve mean arterial pressure of 30-55 mmHg. Deep hypothermic circulatory arrest (DHCA) to $18^{\circ} \mathrm{C}$ was used at the discretion of the surgeon. Modified ultrafiltration was performed in infants and patients below $10 \mathrm{~kg}$ after CPB.

\section{Regional Oxygen Saturation Monitoring}

The use of $\mathrm{CrO}_{2}$ and $\mathrm{SrO}_{2}$ in patients undergoing cardiac surgery is not routine at our center. During the study, $\mathrm{CrO}_{2}$ and $\mathrm{SrO}_{2}$ were measured in the context of a prospective observational study and not for clinical purposes.

Regional oxygen saturation was measured using the Medtronic INVOS 5100C, Boulder, CO, USA. Two site recording from $\mathrm{CrO}_{2}$ and $\mathrm{SrO}_{2}$ regions were performed before induction of anesthesia. The cerebral sensor was placed over the left or right forehead midway between the eyebrow and hairline and the somatic sensor was placed over the left or right flank at T12-L2 vertebral level. After a short period to allow for adjustments to obtain good contact, the baseline tissue oxygenation was recorded. In children who were fretful and uncooperative, the probes were placed and baseline recorded soon after induction of anesthesia. Subsequently, data were collected and downloaded to a storage device every $6 \mathrm{~s}$ throughout the surgery and for $48 \mathrm{~h}$ from admission to the PICU. A pre-discharge recording was made just prior to hospital discharge-this was done over a period of $4-6 \mathrm{~h}$ while the patient was asleep. If the hospital discharge was delayed the pre-discharge recording was taken whenever the attending cardiologist deemed the patient medically fit for discharge.

\section{Data Extraction}

Intraoperative clinical data were extracted from important time-points such as time of induction, median sternotomy, start and end of $\mathrm{CPB}$, aortic clamp and unclamp, and end of surgery. Clinical and laboratory variables (e.g., heart rate, arterial blood pressure, central venous pressure, temperature, urine output, arterial blood gases, and serum lactate) were measured and recorded as per routine practice in the operating theater and PICU. Acute kidney injury was defined according to the RIFLE criteria. Including those who were at Risk of kidney injury $(\geq 1.5 \times$ baseline creatinine or urine output $<0.5 \mathrm{ml} / \mathrm{kg} / \mathrm{h})(13)$. 


\section{Statistical Analysis}

Cerebral oximetry and $\mathrm{SrO}_{2}$ data were captured electronically every $6 \mathrm{~s}$. These were summarized into medians [interquartile ranges (IQR)] for six time-points for comparison: (1) pre-CPB (from time of application of NIRS sensors before induction of anesthesia to time of $\mathrm{CPB}$ initiation); (2) on $\mathrm{CPB}$ (from time of initiation to end $\mathrm{CPB}$ ); (3) post- $\mathrm{CPB}$ (from time of end $\mathrm{CPB}$ to transfer out from operating theater); (4) Day 1 PICU (from time of PICU admission for $24 \mathrm{~h}$ ); (5) Day 2 PICU (from 24 to $48 \mathrm{~h}$ of PICU admission); and (6) discharge (4-6 h overnight prior to day of anticipated discharge). Categorical data were presented as counts and percentages. Continuous data were presented as medians and IQR. Differences between categorical variables were analyzed using the Fisher's exact test. Differences between continuous variables were analyzed using the Wilcoxon's signed rank test. Statistical analysis was performed using SAS 9.4 for Windows (SAS Institute Cary, NC, USA). All statistical tests were two-tailed, and the significance level was taken as $p<0.05$.

\section{RESULTS}

A total of 24 children with cyanotic CHD underwent surgery over the 1-year study period and all were approached for consent. Parents of two children declined to participate in the study and parents of another child withdrew consent after enrollment (Figure 1). The overall median age was $18.1(7.8,51.4)$ months (Table 1). Fifteen [15/21 (71.4\%)] children underwent corrective surgeries. The most common surgery was repair of tetralogy of Fallot [7/21 (33.3\%)] followed by the Fontan procedure [3/21 (14.3\%)] (Table 2). The median CPB and aortic cross-clamp times were $187.5(113.0,263.5)$ and $80.0(37.0,133.0)$ minutes, respectively (Table 2). Only one patient (Norwood procedure) required total circulatory arrest (duration $77 \mathrm{~min}$ ). Intraoperatively, adrenaline [20/21 (95.2\%)] and milrinone [18/21 (85.7\%)] were the most frequently used inotropes.
All patients remained on mechanical ventilation on arrival to the PICU. Five [5/21 (23.8\%)] were extubated within several hours of admission and 9/21 (42.9\%) on postoperative day 1 . The median duration of mechanical ventilation was 21.0 (13.0, 92.0) hours. Twenty [20/21 (95.3\%)] patients required inotropic support on PICU admission. The most common inotrope used was milrinone $[18 / 20(90.0 \%)]$, followed by adrenaline [17/20 (85.0\%)] (Table 3). Median lactate levels within the first $24 \mathrm{~h}$ was $2.7(2.3,5.0) \mathrm{mmol} / \mathrm{L}$. Significant hemodynamic events occurred in $17 / 21(80.9 \%)$ patients with the most common being hypotension [14/21 (66.7\%)] (Table 4). Fluid bolus was required more frequently in the corrective surgery group in the first day of PICU compared to the second day [12/15 $(80.0 \%)$ vs. $4 / 15$ (33.3\%); $p=0.022]$. Acute kidney injury occurred in $17 / 21(81.0 \%)$ patients. However, only one $(5.6 \%)$ patient required renal replacement therapy. 3/21 patients required veno-arterial extracorporeal membrane oxygenation postoperatively, of whom 1/3 (33.3\%) survived. Overall PICU mortality was $2 / 21(9.5 \%)$. The causes of death in these two patients were necrotizing enterocolitis and hypoplastic left heart syndrome.

In the corrective surgery group, $\mathrm{SpO}_{2}$ increased immediately post-CPB compared to pre-CPB $[99(98,100)$ vs. $86 \%(79,90)$; $p<0.001]$ and remained in the normal range through to hospital discharge $[97 \%(92,98)]$ (Table 5). Post-CPB $\mathrm{CrO}_{2}$ did not change from pre-CPB $[72.8(58.8,79.0)$ vs. $72.1 \%(63.0,78.3)$; $p=0.761]$. Postoperative day $2 \mathrm{CrO}_{2}$ was also not different from pre-CPB [68.3 (55.0, 74.4) vs. 72.1\% (63.0, 78.3); $p=0.520]$. On hospital discharge, however, $\mathrm{CrO}_{2}$ was lower than baseline (pre-CPB) [60.5 $(53.6,62.9)$ vs. $72.1 \%(63.0,78.3) ; p=0.005]$. Of note, the majority of our patients $[11 / 21(52.4 \%)]$ had their baseline (pre-CPB) taken a few minutes after intubation and ventilation.

Post-CPB $\mathrm{SrO}_{2}$ increased compared to pre-CPB $[87.3$ $(77.2,89.5)$ vs. $72.7 \%(65.6,77.3) ; p<0.001]$ but progressively

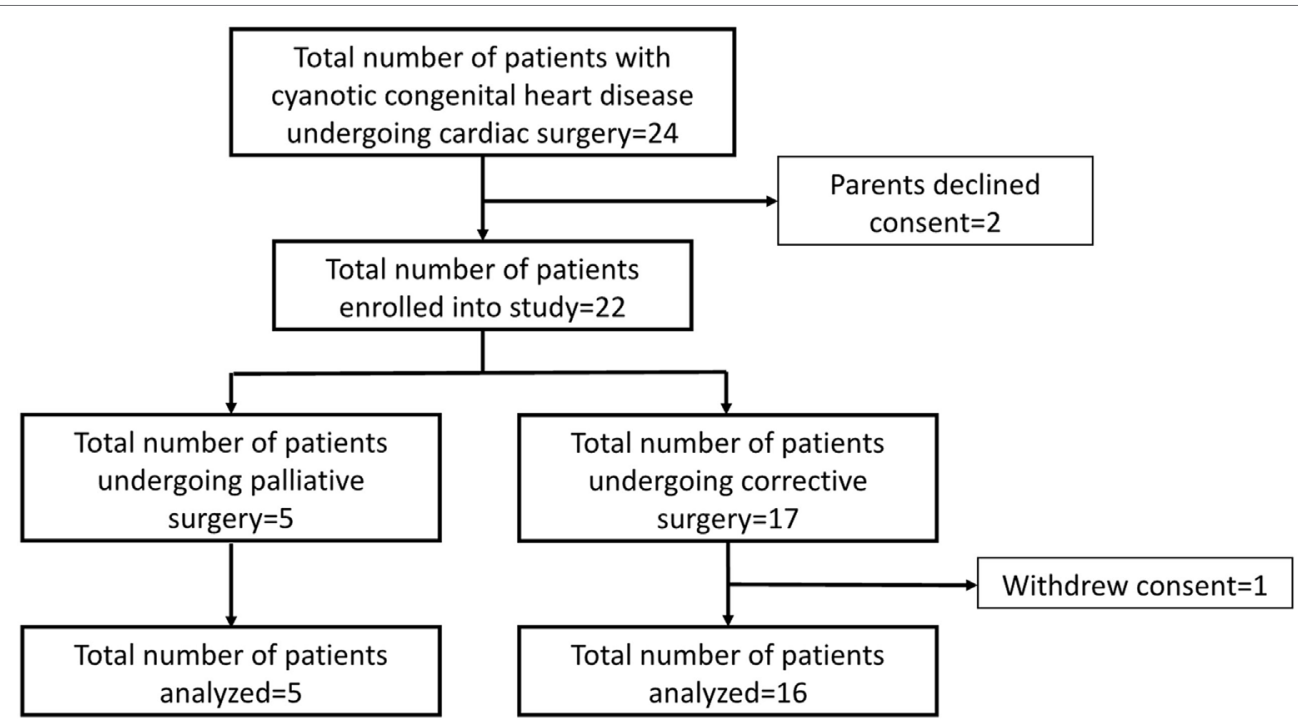

FIGURE 1 | CONSORT diagram. 
TABLE 1 | Summary of patient characteristics $(N=21)$.

\begin{tabular}{|c|c|c|c|}
\hline Patient characteristics & Corrective surgery $(N=15)$ & Non-corrective surgery $(N=6)$ & All patients $(N=21)$ \\
\hline Age, months & $18.7(10.3,51.4)$ & $8.0(0.4,46.0)$ & $18.1(7.8,51.4)$ \\
\hline Weight, kg & $10.5(7.0,14.1)$ & $6.0(3.0,11.4)$ & $8.5(5.4,13.5)$ \\
\hline Male gender & $6(40.0)$ & $3(50.0)$ & $9(42.9)$ \\
\hline \multicolumn{4}{|l|}{ Ethnicity } \\
\hline Chinese & 8 (53.3) & $3(50.0)$ & $11(52.4)$ \\
\hline Malay & $1(6.7)$ & $2(33.3)$ & $3(14.3)$ \\
\hline Indian & $1(6.7)$ & $0(0.0)$ & $1(4.8)$ \\
\hline Others & 5 (33.3) & $1(16.7)$ & $6(28.6)$ \\
\hline Comorbidities & $3(20.0)$ & 2 (33.3) & $5(23.8)$ \\
\hline \multicolumn{4}{|l|}{ RACHS score } \\
\hline 1 & $0(0)$ & $0(0)$ & $0(0)$ \\
\hline 2 & $9(60.0)$ & $4(66.7)$ & $13(61.9)$ \\
\hline 3 & $6(40.0)$ & $0(0)$ & $6(28.6)$ \\
\hline 4 & $0(0)$ & $1(16.7)$ & $1(4.8)$ \\
\hline 5 & $0(0)$ & $0(0)$ & $0(0)$ \\
\hline 6 & $0(0)$ & $1(16.7)$ & $1(4.8)$ \\
\hline \multicolumn{4}{|l|}{ STAT category } \\
\hline 1 & $0(0.0)$ & $0(0.0)$ & $0(0.0)$ \\
\hline 2 & $12(80.0)$ & $3(50.0)$ & $15(71.4)$ \\
\hline 3 & $2(13.3)$ & $1(16.7)$ & $3(14.3)$ \\
\hline 4 & $1(6.7)$ & $1(16.7)$ & $2(9.5)$ \\
\hline 5 & $0(0.0)$ & $1(16.7)$ & $1(4.8)$ \\
\hline PIM 3 & $0.1(0.1,0.2)$ & $0.2(0.1,0.7)$ & $0.1(0.1,0.2)$ \\
\hline PELOD 2 & $7.0(5.0,11.0)$ & $5.5(2.0,9.0)$ & $7.0(5.0,10.0)$ \\
\hline Cardiopulmonary bypass time, min & $193.0(134.0,269.0)$ & $121.0(82.0,243.0)$ & $187.5(113.0,263.5)$ \\
\hline Aortic cross-clamp time, min & $110.0(65.0,171.0)$ & $33.0(27.0,34.0)$ & $80.0(37.0,133.0)$ \\
\hline Duration of surgery, min & $335.0(281.0,428.0)$ & $360.5(263.0,497.0)$ & $354.0(281.0,428.0)$ \\
\hline Intraoperative adrenaline & $14(93.3)$ & $6(100.0)$ & $20(95.2)$ \\
\hline Intraoperative milrinone & 14 (93.3) & $4(66.7)$ & $18(85.7)$ \\
\hline Acute kidney injury & $12(80.0)$ & 5 (83.3) & $17(81.0)$ \\
\hline PICU mortality & $1(6.7)$ & $1(16.7)$ & $2(9.5)$ \\
\hline ECMO & $1(6.7)$ & 2 (33.3) & $3(14.3)$ \\
\hline Length of PICU stay, days & $2.0(1.0,6.0)$ & $2.0(1.0,7.0)$ & $2.0(1.0,6.0)$ \\
\hline Length of hospital stay, days & $10.0(8.0,21.0)$ & $12.0(5.0,100.0)$ & $10.0(8.0,21.0)$ \\
\hline
\end{tabular}

Categorical and continuous variables presented as counts (percentages) and median (interquartile range), respectively.

PELOD, pediatric logistic organ dysfunction; PIM, Pediatric Index of Mortality; PICU, pediatric intensive care unit; RACHS, risk adjustment for congenital heart surgery, STAT, Society of Thoracic Surgeons-European Association for Cardio-Thoracic Surgery; ECMO, extracorporeal membrane oxygenation.

decreased during PICU stay to a value lower than baseline at hospital discharge $[66.9(57.3,76.9)$ vs. $72.7 \%(65.6,77.3)$; $p=0.048]$. The aggregate $\mathrm{SpO}_{2}, \mathrm{CrO}_{2}$, and $\mathrm{SrO}_{2}$ trend for patients who underwent corrective surgery are shown in Figures 2-4. There were no significant changes in $\mathrm{SpO}_{2}, \mathrm{CrO}_{2}$, and $\mathrm{SrO}_{2}$ in the palliative surgery group from baseline, postoperatively and on discharge.

\section{DISCUSSION}

We prospectively studied a small cohort of 21 children with cyanotic CHD and characterized their $\mathrm{CrO}_{2}$ and $\mathrm{SrO}_{2}$ longitudinally over the perioperative period. Unlike $\mathrm{SpO}_{2}$ which normalized immediately after corrective surgery, $\mathrm{CrO}_{2}$ did not increase after surgical correction of cyanotic CHD even up to the time of hospital discharge. $\mathrm{SrO}_{2}$ showed a transient increment during and after CPB but decreased subsequently.

Arterial oxygen saturation measured by $\mathrm{SpO}_{2}$ increases after surgical correction of cyanotic $\mathrm{CHD}$. $\mathrm{CrO}_{2}$, measuring the concentration of hemoglobin and oxy-hemoglobin in arterioles, capillaries and venules reflects cerebral tissue oxygenation which is taken as a surrogate of cerebral venous oxygenation $\left(\mathrm{SvO}_{2}\right)$ $(14,15)$. In this observational study, the $\mathrm{CrO}_{2}$ remains the same. This seems to suggest that cerebral fractional oxygen extraction $(\mathrm{cFOE})\left[\mathrm{cFOE}=\left(\mathrm{SaO}_{2}-\mathrm{SvO}_{2}\right) / \mathrm{SaO}_{2}\right]$ increased during the post-surgical repair period (16). These data are consistent with the literature which describes increased cerebral metabolic rate of oxygen $\left(\mathrm{CRMO}_{2}\right)$ post-cardiac surgery (17). An observational study in term neonates with the primary objective of demonstrating $\mathrm{CRMO}_{2}$, arterial oxygen content, cerebral blood flow, and $\mathrm{cFOE}$ differences due to $\mathrm{CPB}$ duration and DHCA, reported increased $\mathrm{CRMO}_{2}$ [increase of 121\% (59-207) from baseline; $p=0.17$ ] and cFOE [increase of $145 \%(107,222)$ from baseline; $p=0.005$ ] $12 \mathrm{~h}$ after returning from the operating room (17). Taking these findings together, this may suggest that we should not target $\mathrm{CrO}_{2}$ above baseline after surgical correction. Of note, in the current study, only one patient underwent DHCA, which is associated with decreased CRMO and cFOE 
TABLE 2

Summary of cardiac diagnosis and surgical procedures $(N=21)$.

\begin{tabular}{|c|c|c|c|c|}
\hline Patient & Cardiac lesions & Surgical procedures & Corrective & Survived \\
\hline 1 & $\begin{array}{l}\text { Pulmonary atresia with ventricular septal defect, major } \\
\text { aortopulmonary collaterals, left lower pulmonary artery stenosis }\end{array}$ & $\begin{array}{l}\text { Repair of pulmonary artery stenosis (left main pulmonary } \\
\text { artery to left lower pulmonary artery graft) }\end{array}$ & No & Yes \\
\hline 2 & $\begin{array}{l}\text { Hypoplastic right ventricle, large atrial and ventricular septal defect, } \\
\text { transposition of the great arteries }\end{array}$ & Fontan procedure & Yes & Yes \\
\hline 3 & Tetralogy of Fallot & Total repair & Yes & Yes \\
\hline 4 & Pulmonary atresia with ventricular septal defect & $\begin{array}{l}\text { Right ventricle to pulmonary artery conduit } \\
\text { Ventricular septal defect repair }\end{array}$ & Yes & Yes \\
\hline 5 & Tetralogy of Fallot & Total repair & Yes & Yes \\
\hline 7 & Tetralogy of Fallot & Total repair & Yes & Yes \\
\hline 8 & $\begin{array}{l}\text { Dextrocardia, hypoplastic right ventricle, transposition of the great } \\
\text { arteries, severe pulmonary stenosis, moderate atrial septal defect }\end{array}$ & Fontan procedure & Yes & Yes \\
\hline 9 & Tetralogy of Fallot & Total repair & Yes & Yes \\
\hline 10 & Tetralogy of Fallot & Total repair & Yes & Yes \\
\hline 11 & $\begin{array}{l}\text { Pulmonary atresia intact ventricular septum, patent ductus } \\
\text { arteriosus }\end{array}$ & $\begin{array}{l}\text { Right ventricular outflow tract augmentation } \\
\text { Ligation of patent ductus arteriosus }\end{array}$ & Yes & Yes \\
\hline 12 & $\begin{array}{l}\text { Complete atrioventricular septal defect, hypoplastic aorta, } \\
\text { coarctation of the aorta }\end{array}$ & Coarctation repair within 30 days & No & Yes \\
\hline 13 & $\begin{array}{l}\text { Pulmonary atresia with intact ventricular septum, moderate mitral } \\
\text { regurgitation }\end{array}$ & $\begin{array}{l}\text { Fontan procedure } \\
\text { Mitral annuloplasty }\end{array}$ & Yes & Yes \\
\hline 14 & $\begin{array}{l}\text { Congenitally corrected transposition of the great arteries, large } \\
\text { ventricular septal defect, severe pulmonary stenosis }\end{array}$ & $\begin{array}{l}\text { Right ventricle to pulmonary artery conduit } \\
\text { Ventricular septal defect repair }\end{array}$ & Yes & Yes \\
\hline 15 & Tetralogy of Fallot & Total repair & Yes & Yes \\
\hline 16 & $\begin{array}{l}\text { Complete atrioventricular septal defect with moderate to severe } \\
\text { atrioventricular valve regurgitation, total anomalous pulmonary } \\
\text { venous drainage }\end{array}$ & $\begin{array}{l}\text { Repair of complete atrioventricular canal } \\
\text { Repair of total anomalous pulmonary venous drainage }\end{array}$ & Yes & No \\
\hline 17 & $\begin{array}{l}\text { Tricuspid valve atresia, hypoplastic right ventricle, ventricular septal } \\
\text { defect }\end{array}$ & Bidirectional Glenn & No & Yes \\
\hline 18 & $\begin{array}{l}\text { Atrioventricular septal defect, hypoplastic right ventricle, pulmonary } \\
\text { atresia, major aortopulmonary collaterals }\end{array}$ & $\begin{array}{l}\text { Unifocalization for pulmonary atresia (right lower and middle } \\
\text { major aortopulmonary collateral artery unifocalization) } \\
\text { Systemic to pulmonary artery shunt (modified Blalock Taussig } \\
\text { shunt) }\end{array}$ & No & No \\
\hline 19 & Tetralogy of Fallot & Total repair & Yes & Yes \\
\hline 20 & $\begin{array}{l}\text { Hypoplastic right ventricle, large ventricular septal defect, } \\
\text { pulmonary stenosis, transposition of the great arteries }\end{array}$ & Bidirectional Glenn & No & Yes \\
\hline 21 & $\begin{array}{l}\text { Hypoplastic left heart syndrome, partial anomalous pulmonary } \\
\text { venous drainage }\end{array}$ & $\begin{array}{l}\text { Stage } 1 \text { repair of hypoplastic left heart syndrome } \\
\text { (Norwood operation) }\end{array}$ & No & No \\
\hline 22 & Double outlet right ventricle-Tetralogy of Fallot type & Repair of double outlet right ventricle & Yes & Yes \\
\hline
\end{tabular}

(18). Moreover, this patient died and did not contribute to hospital discharge $\mathrm{CrO}_{2}$ data. To the best of our knowledge, there are no studies describing $\mathrm{cFOE}$ trends in the postoperative period beyond the first postoperative day in children with CHD. As we were not able to measure cFOE directly, future studies describing these values in children undergoing cardiac surgery would be required to describe this physiological response in greater detail.

Baseline $\mathrm{CrO}_{2}$ and $\mathrm{SrO}_{2}$ in our cohort were 63.8 (57.2, 76.6) and $74.6 \%(68.9,82.4)$, respectively. These values are relatively high compared to other studies involving children with cyanotic CHD. A large prospective study $(n=112)$ reported different baseline $\mathrm{CrO}_{2}$ with different diagnosis: tetralogy of Fallot $(57 \pm 12)$, pulmonary atresia $(38 \pm 6)$, single ventricle with aortopulmonary shunt (50 \pm 7 ), bidirectional cavo-pulmonary connection $(43 \pm 6)$, and Fontan $(70 \pm 4)$. All patients except those in the Fontan group had $\mathrm{CrO}_{2}$ lower than the control group of healthy patients $(68 \pm 10)(10)$. In staged surgery for single-ventricle physiology, patients had a baseline $\mathrm{CrO}_{2}$ of $61 \%$ at pre-stage 1 , $48 \%$ post-stage $1, \mathrm{CrO}_{2}$ of $42 \%$ pre-stage $2,53 \%$ post-stage 2 , and $\mathrm{CrO}_{2}$ of $70 \%$ pre-Fontan which remained the same after completion of the Fontan procedure (11). We postulate the reason for the high baseline in our study is because the majority of our patients had their baseline (pre-CPB) taken a few minutes after intubation and ventilation. Patients in this cohort were mostly toddlers and young children who were uncooperative for placement of NIRS sensors prior to anesthesia. Hence, we postulate the high baseline tissue oximetry readings were because the patients were already 
TABLE 3 | Summary of intensive care unit (ICU) variables $(N=21)$.

\begin{tabular}{|c|c|c|c|c|c|c|}
\hline \multirow[t]{2}{*}{ ICU variables } & \multicolumn{3}{|c|}{ Corrective surgery $(N=15)$} & \multicolumn{3}{|c|}{ Non-corrective surgery $(N=6)$} \\
\hline & Day $1(N=15)$ & Day $2(N=12)$ & $p$-value & Day $1(N=6)$ & Day $2(N=3)$ & $p$-value \\
\hline Toe-core gap, ${ }^{\circ} \mathrm{C}$ & $6.2(4.0,8.2)$ & $6.7(4.3,7.8)$ & 0.470 & $2.8(2.8,3.0)$ & $5.8(3.7,8.0)$ & 1.000 \\
\hline Central venous pressure, $\mathrm{mmHg}$ & $12.3(11.2,13.0)$ & $14.0(8.0,15.3)$ & 0.278 & $8.5(6.0,10.4)$ & $11.5(9.3,19.9)$ & 0.500 \\
\hline Lactate, $\mathrm{mmol} / \mathrm{L}$ & $2.7(2.3,5.0)$ & $1.9(1.4,3.7)$ & 0.193 & $2.4(1.3,5.5)$ & $2.0(1.1,3.8)$ & 0.250 \\
\hline Hemoglobin, g/dL & $13.0(12.2,14.5)$ & $11.5(10.7,13.6)$ & 0.148 & $11.6(10.7,12.6)$ & $12.9(10.1,14.0)$ & 1.000 \\
\hline Urine output, ml/kg/h & $0.8(0.6,1.5)$ & $1.1(0.9,2.2)$ & 0.077 & $1.8(1.1,2.0)$ & $1.2(0.3,4.1)$ & 1.000 \\
\hline Cumulative balance, $\mathrm{ml} / \mathrm{kg}$ & $0.5(-9.1,10.9)$ & $0.6(-4.3,19.1)$ & 0.791 & $4.3(-0.1,115.7)^{\mathrm{a}}$ & $93.7(4.8,130.5)^{\mathrm{a}}$ & 1.000 \\
\hline \multicolumn{7}{|l|}{ Postoperative inotrope } \\
\hline Dopamine & $0(0.0)$ & $0(0.0)$ & - & $1(16.7)$ & 1 (33.3) & 1.000 \\
\hline Adrenaline & $14(93.3)$ & $6(50.0)$ & 0.024 & $3(50.0)$ & $2(66.7)$ & 1.000 \\
\hline Noradrenaline & 2 (13.3) & $3(5.0)$ & 0.628 & $1(16.7)$ & $2(66.7)$ & 0.226 \\
\hline Milrinone & $15(100.0)$ & $10(83.3)$ & 0.188 & $3(50.0)$ & $2(66.7)$ & 1.000 \\
\hline Phentolamine & $0(0.0)$ & $0(0.0)$ & - & $1(16.7)$ & 1 (33.3) & 1.000 \\
\hline \multicolumn{7}{|l|}{ Ventilation variables } \\
\hline $\mathrm{PIP}, \mathrm{cm} \mathrm{H} \mathrm{H}_{2} \mathrm{O}$ & $17.2(13.2,19.3)$ & $20.6(19.0,21.3)$ & 0.688 & $18.0(16.9,20.3)$ & $20.0(18.0,22.0)$ & 1.000 \\
\hline PEEP, $\mathrm{cm} \mathrm{H}_{2} \mathrm{O}$ & $5.0(5.0,6.0)$ & $6.3(5.0,7.0)$ & 1.000 & $6.5(5.0,8.5)$ & $8.0(5.0,10.0)$ & 1.000 \\
\hline MAP, $\mathrm{cm} \mathrm{H}_{2} \mathrm{O}$ & $8.5(7.4,9.8)$ & $10.9(10.3,11.4)$ & 0.438 & $8.9(8.5,10.8)$ & $10.4(8.8,12.0)$ & 0.500 \\
\hline $\mathrm{TV}, \mathrm{mL} / \mathrm{kg}$ & $7.9(6.8,10.2)$ & $6.6(6.3,6.8)$ & 0.156 & $6.0(4.1,7.5)$ & $3.7(2.2,5.1)$ & 1.000 \\
\hline $\mathrm{FiO}_{2}, \%$ & $0.4(0.3,0.7)$ & $0.4(0.3,0.5)$ & 0.734 & $0.3(0.2,0.4)$ & $0.2(0.2,0.3)$ & 0.500 \\
\hline $\mathrm{SpO}_{2}, \%$ & $97(95,98)$ & $98(89,99)$ & 0.266 & $80(77,92)$ & $90(70,98)$ & 0.250 \\
\hline Inhaled nitric oxide & $1(6.7)$ & $1(8.3)$ & 1.000 & $0(0.0)$ & $0(0.0)$ & - \\
\hline \multicolumn{7}{|l|}{ Blood gas variables } \\
\hline $\mathrm{pH}$ & $7.4(7.4,7.4)$ & $7.4(7.4,7.4)$ & 0.966 & $7.4(7.3,7.4)$ & $7.4(7.3,7.4)$ & 0.500 \\
\hline $\mathrm{PaO}_{2}, \mathrm{mmHg}$ & $95.0(65.8,104.7)$ & $93.7(52.9,117.6)$ & 0.898 & $48.6(44.4,50.8)$ & $77.2(48.0,100.1)$ & 0.750 \\
\hline $\mathrm{PaCO}_{2}, \mathrm{mmHg}$ & $39.1(34.8,40.8)$ & $37.5(34.6,39.6)$ & 0.520 & $44.4(42.2,48.1)$ & $41.8(39.8,46.2)$ & 0.250 \\
\hline $\mathrm{HCO}_{3}, \mathrm{mmol} / \mathrm{L}$ & $22.8(21.8,23.4)$ & $22.6(21.5,23.3)$ & 0.465 & $24.0(22.0,25.5)$ & $23.8(20.0,27.5)$ & 1.000 \\
\hline Base deficit & $-2.3(-3.6,-1.6)$ & $-2.9(-4.1,-2.0)$ & 0.365 & $-0.1(-2.8,0.9)$ & $-1.3(-5.0,4.3)$ & 1.000 \\
\hline
\end{tabular}

Categorical and continuous variables presented as counts (percentages) and median (interquartile range), respectively.

aHigh third quartile due to two patients (number 18 and 21) with high cumulative positive balance.

PIP, peak inspiratory pressure; $P E E P$, positive end expiratory pressure; $M A P$, mean airway pressure; $T V$, tidal volume; FiO ${ }_{2}$, fraction of inspired oxygen; SpO $\mathrm{O}_{2}$, pulse oximetry;

$\mathrm{PaO}_{2}$, partial pressure of arterial oxygen; $\mathrm{PaCO}_{2}$, partial pressure of carbon dioxide; $\mathrm{HCO}_{3}$, bicarbonate.

Bold value represents $p<0.05$.

TABLE 4 | Significant postoperative hemodynamic events and interventions within first 2 postoperative days.

\begin{tabular}{|c|c|c|c|c|c|c|}
\hline & \multicolumn{3}{|c|}{ Corrective surgery $(N=15)$} & \multicolumn{3}{|c|}{ Non-corrective surgery $(N=6)$} \\
\hline & $\begin{array}{c}\text { Day } 1(N=15) \\
n(\%) n E\end{array}$ & $\begin{array}{c}\text { Day } 2(N=12) \\
n(\%) n E\end{array}$ & $p$-value & $\begin{array}{c}\text { Day } 1(N=6) \\
n(\%) n E\end{array}$ & $\begin{array}{c}\text { Day } 2(N=3) \\
n(\%) n E\end{array}$ & $p$-value \\
\hline \multicolumn{7}{|l|}{ Hemodynamic events } \\
\hline Hypotension & 11 (73.3) 62 & $5(41.7) 53$ & 0.130 & $3(50.0) 47$ & $2(66.7) 33$ & 1.000 \\
\hline Desaturation & $1(6.7) 1$ & $1(8.3) 1$ & 1.000 & 2 (33.3) 10 & 1 (33.3) 21 & 1.000 \\
\hline Cannula dislodgement & $1(6.7) 17$ & $0(0.0) 0$ & 1.000 & $1(16.7) 1$ & $1(33.3) 1$ & 1.000 \\
\hline Arrhythmia & $7(46.7) 54$ & 4 (33.3) 41 & 0.696 & $1(16.7) 17$ & $0(0.0) 0$ & 1.000 \\
\hline Cardiac arrest & $0(0.0) 0$ & $0(0.0) 0$ & - & $0(0.0) 0$ & $0(0.0) 0$ & - \\
\hline Hemorrhage & $3(20.0) 9$ & $2(16.7) 3$ & 1.000 & $0(0.0) 0$ & $0(0.0) 0$ & - \\
\hline Oliguria & $1(6.7) 2$ & $0(0.0) 0$ & 1.000 & $0(0.0) 0$ & $0(0.0) 0$ & - \\
\hline \multicolumn{7}{|l|}{ Interventions } \\
\hline Fluid bolus & 12 (80.0) 51 & 4 (33.3) 21 & 0.022 & 2 (33.3) 17 & 1 (33.3) 5 & 1.000 \\
\hline Transfusion & 5 (33.3) 19 & $2(16.7) 3$ & 0.408 & $3(50.0) 31$ & $2(66.7) 13$ & 1.000 \\
\hline Increase inotrope & $3(20.0) 6$ & $3(25.0) 4$ & 1.000 & $1(16.7) 2$ & $2(66.7) 7$ & 0.226 \\
\hline Cardiac massage & $0(0.0) 0$ & $0(0.0) 0$ & - & $0(0.0) 0$ & $0(0.0) 0$ & - \\
\hline Defirbillation/cardioversion & $0(0.0) 0$ & $0(0.0) 0$ & - & $0(0.0) 0$ & $0(0.0) 0$ & - \\
\hline ECMO & $0(0.0) 0$ & $0(0.0) 0$ & - & $1(16.7) 25$ & 1 (33.3) 24 & 1.000 \\
\hline Amiodarone & $1(6.7) 1$ & $1(8.3) 11$ & 1.000 & $0(0.0) 0$ & $1(33.3) 1$ & 0.333 \\
\hline Chest toilet & $1(6.7) 1$ & $0(0.0) 0$ & 1.000 & $0(0.0) 0$ & $0(0.0) 0$ & - \\
\hline Pacing & $3(20.0) 28$ & $2(16.7) 25$ & 1.000 & $1(16.7) 8$ & $0(0.0) 0$ & 1.000 \\
\hline
\end{tabular}

Categorical and continuous variables presented as counts (percentages) and median (interquartile range), respectively.

ECMO, extracorporeal membrane oxygenation; $n$, number of subjects (\% = percent of subjects); $n E$, total number events.

Bold value represents $p<0.05$. 


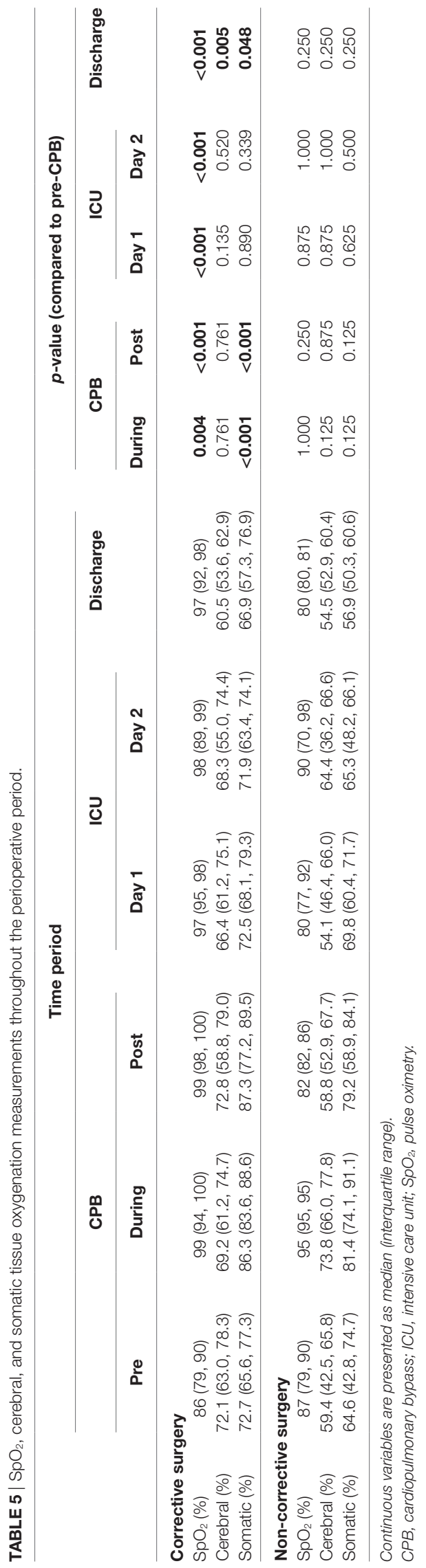

sedated, intubated, and ventilated, and this may lead to increase in $\mathrm{CrO}_{2}$ and $\mathrm{SrO}_{2}$ readings $(19,20)$.

A retrospective study $(n=25)$ of infants undergoing elective cardiac surgery showed that $\mathrm{CrO}_{2}$ was always lower than that of other regions (including renal, arm, and thigh) (21). This reflects a higher FOE across the cerebral bed compared with the somatic bed (22). We observed a similar phenomenon in our cohort, and this supports the use of $\mathrm{CrO}_{2}$ as the main site for regional oximetry monitoring in the most vulnerable of patients. These patients while on $\mathrm{CPB}$ also showed a higher $\mathrm{CrO}_{2}$ and $\mathrm{SrO}_{2}$ compared to other perioperative time-points. This increase associated with CPB was observed regardless of anatomical diagnosis $(10,11$, 23). In our study, we noted that there was an increase in both cerebral and $\mathrm{SrO}_{2}$ sites for on $\mathrm{CPB}$. $\mathrm{SrO}_{2}$ was higher during $\mathrm{CPB}$ compared to pre-CPB $[86.3(83.6,88.6)$ vs. $72.7 \%(65.6,77.3)$; $p<0.001]$. This effect normalized after coming off CPB. It is suggested that the marked increase in $\mathrm{SrO}_{2}$ site (above normal levels) is due to over-oxygenation or reduced oxygen consumption at these sites (21).

A trend of declining $\mathrm{SrO}_{2}$ is observed over the first $48 \mathrm{~h}$ as the patient recovers from surgery in the PICU. Although we were not able to elicit the exact reasons for this phenomenon in this study, this decreasing trend may be explained by factors that affect $\mathrm{CrO}_{2}$ and $\mathrm{SrO}_{2}$ such as increased oxygen demand after surgery as the patient is weaned from sedation and ventilation, low cardiac output states, hypovolemia, microcirculatory disturbance, increased vascular tone, and changes in the toe-core temperature gap $(5,19,20)$. However, the lack of decline in $\mathrm{CrO}_{2}$ values may be related to the preservation of cerebral autoregulation $(24,25)$.

There was a decrease in both $\mathrm{CrO}_{2}$ and $\mathrm{SrO}_{2}$ at hospital discharge compared to baseline in both the corrective and palliative surgery groups, but it did not reach statistical significance for the latter possibly due to the small sample size. The oximetry readings taken at hospital discharge spanned 4-6 h during overnight sleep when the patient was considered well and fit for home. It is likely the $\mathrm{CrO}_{2}$ and $\mathrm{SrO}_{2}$ at hospital discharge reflects the true baseline and the pre- $\mathrm{CPB}$ values reflect a hyperoxygenated state as it was taken with the patient on mechanical ventilation.

Although this is a pilot and purely descriptive study, to the best of our knowledge, this is the first study to longitudinally examine NIRS perioperatively up to hospital discharge in children with cyanotic $\mathrm{CHD}$ undergoing surgical correction. We demonstrated that $\mathrm{CrO}_{2}$ and $\mathrm{SrO}_{2}$ did not show a sustained increase even up until the time of hospital discharge in patients who had undergone complete repair. Hence, our findings potentially imply that during the postoperative period, oximetry values may not be necessarily higher than baseline even when $\mathrm{SpO}_{2}$ completely normalizes. Even though our sample size was small, we managed to achieve our aim of describing the postoperative $\mathrm{CrO}_{2}$ and $\mathrm{SrO}_{2}$ values beyond $24 \mathrm{~h}$ after surgery. However, due to the small sample size we were not able to meaningfully stratify $\mathrm{CrO}_{2}$ and $\mathrm{SrO}_{2}$ according to baseline characteristics (e.g., age, type of procedure). Another factor may have limited our analysis was that our patients were young and too fretful to have the NIRS sensors placed pre-induction of anesthesia. Most of these sensors were placed after sedation and successful intubation, leading to higher baseline oximetry than expected. Another limitation is the wide 


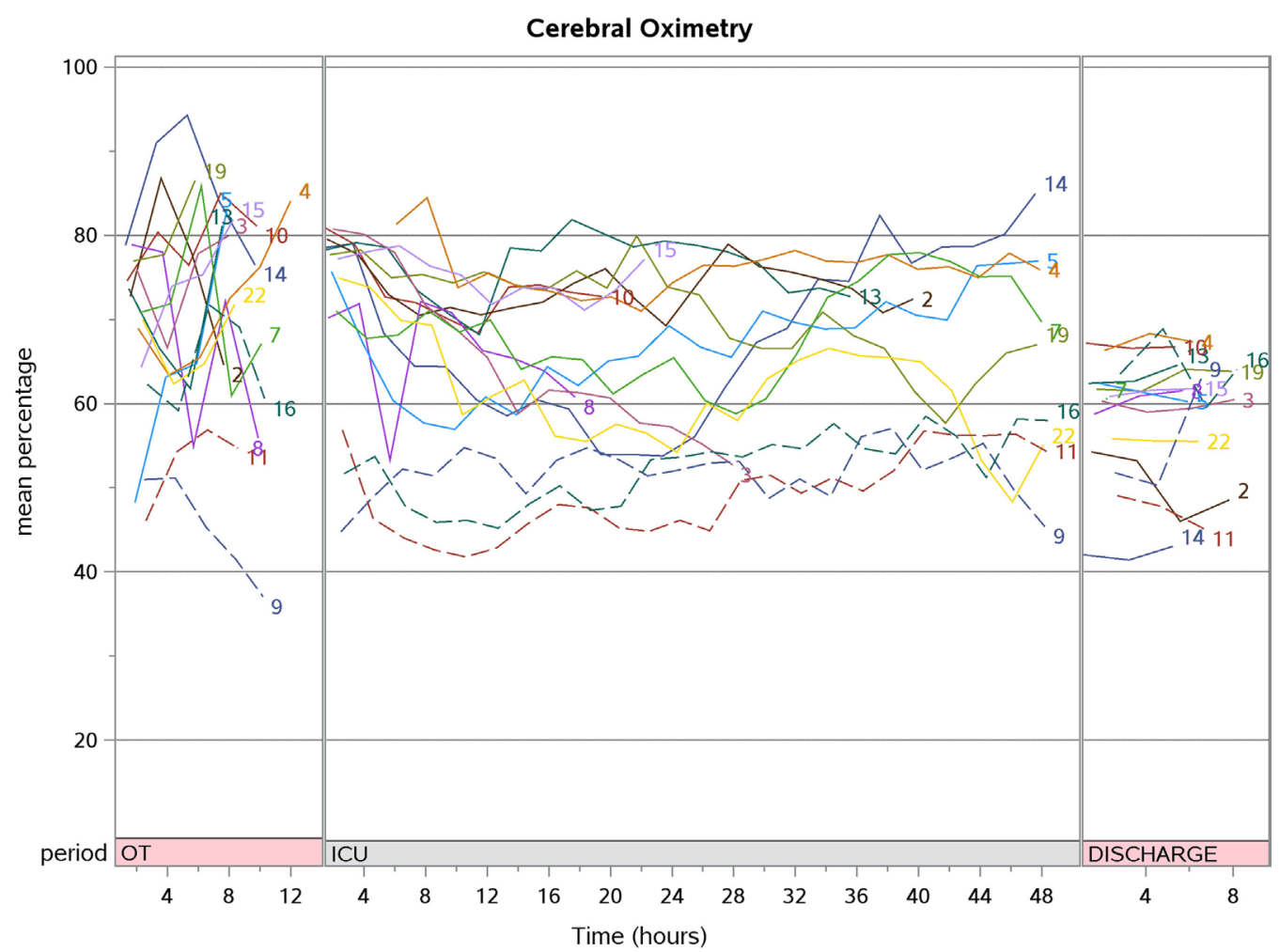

FIGURE 2 | The aggregate cerebral oximetry trend for patients who underwent corrective surgery $(N=15)$.

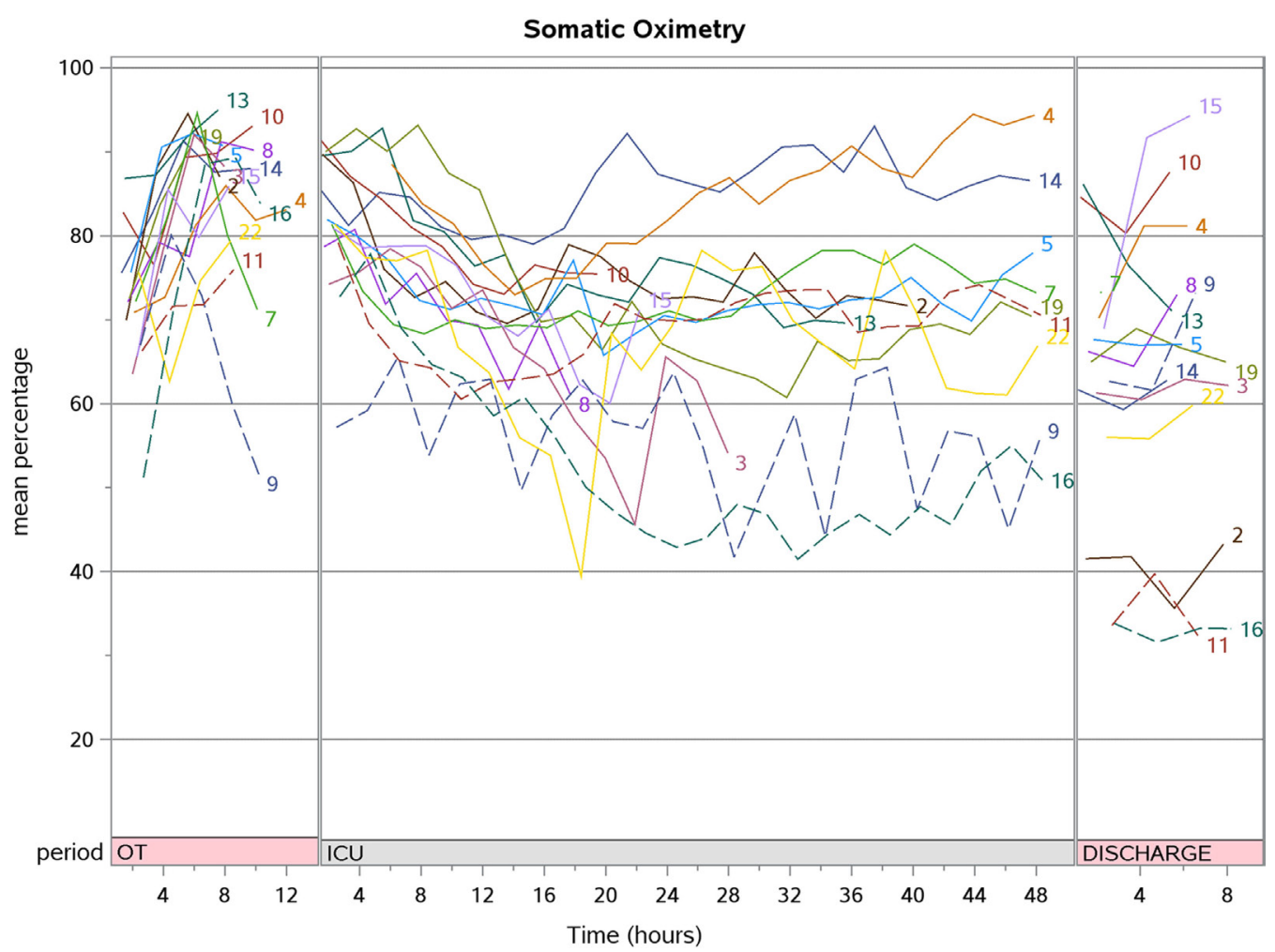

FIGURE 3 | The aggregate somatic oximetry trend for patients who underwent corrective surgery $(N=15)$. 


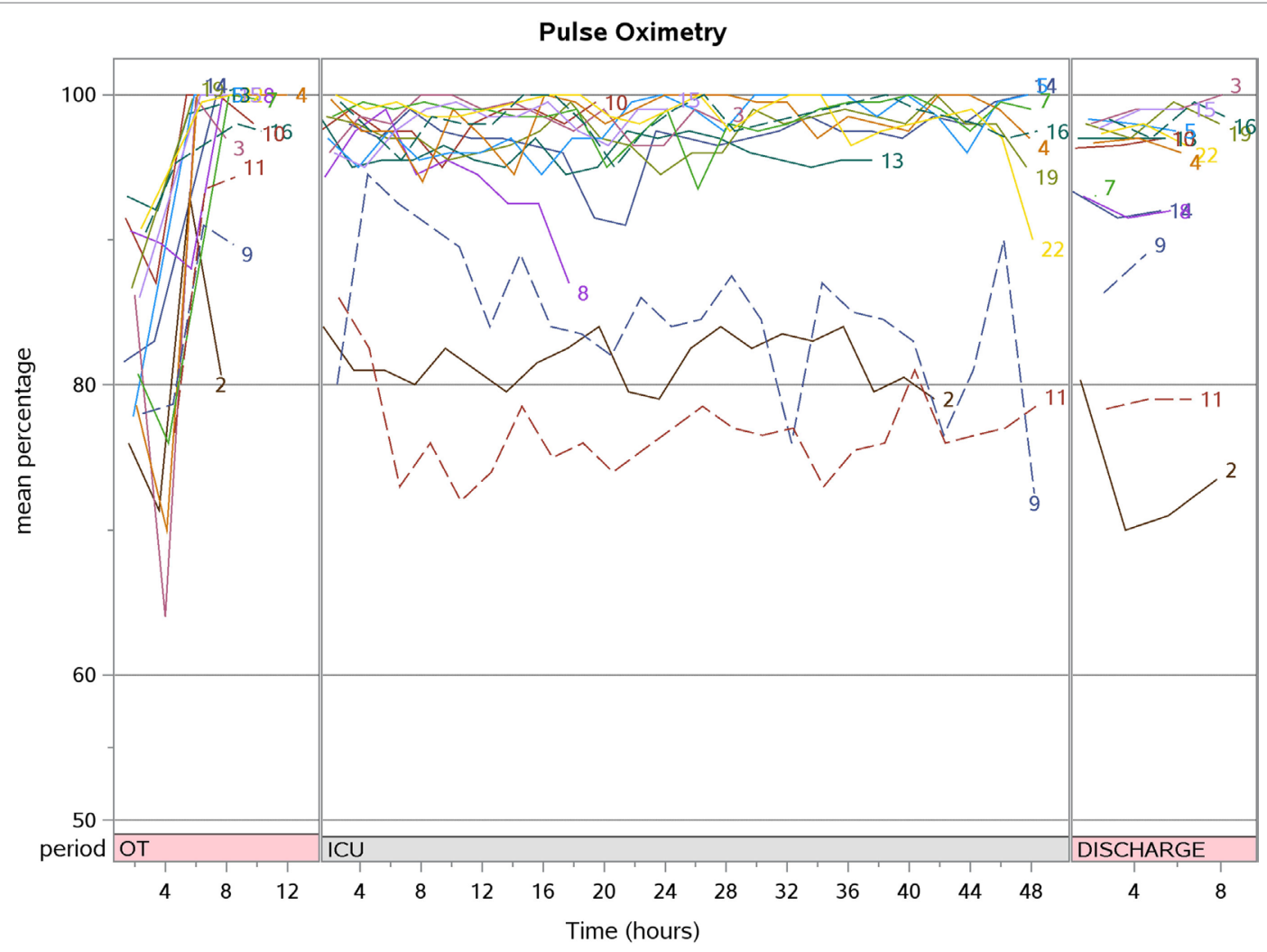

FIGURE $4 \mid$ The aggregate pulse oximetry trend for patients who underwent corrective surgery $(N=15)$.

limits of agreement between one cerebral oximeter and another, making devices non-interchangeable and hence, the results of this study may not be generalized to all makes of the cerebral oximeter (26). Finally, we were not able to obtain NIRS value during longer follow-up period such as 3-6 months after surgery, as we focused our study on the immediate perioperative period.

In conclusion, this pilot study demonstrated that $\mathrm{SpO}_{2}$ normalizes immediately after surgery but $\mathrm{CrO}_{2}$ and $\mathrm{SrO}_{2}$ does not show a sustained increase after surgical correction of cyanotic $\mathrm{CHD}$ even up to the time of hospital discharge. Future larger studies are required to validate our findings so as to inform future guidelines and management algorithms using NIRS in this group of children.

\section{ETHICS STATEMENT}

This study was carried out in accordance with the recommendations of the SingHealth Centralised Institutional Review Board (reference number: 2015/2161). The study protocol was approved by the SingHealth Centralised Institutional Review Board. The

\section{REFERENCES}

1. Ghanayem NS, Hoffman GM. Near infrared spectroscopy as a hemodynamic monitor in critical illness. Pediatr Crit Care Med (2016) 17(8 Suppl 1):S201-6. doi:10.1097/PCC.0000000000000780 legal representative of all subjects gave written informed consent in accordance with the Declaration of Helsinki.

\section{AUTHOR CONTRIBUTIONS}

JW, CC, RM, OW, TT, MN, JA, TL, and JL contributed conception and design of the study; RM and JA performed the statistical analysis; JW and JL wrote the first draft of the manuscript; CC, OW, TT, and MN wrote sections of the manuscript. All authors contributed to manuscript revision, read, and approved the submitted version.

\section{ACKNOWLEDGMENTS}

The authors thank Ms. Kathy Liaw Chiew Suan and Ms. Chen Su Qiu (clinical research coordinators) for assisting with patient recruitment and ensuring protocol adherence. The authors also thank the patients and parents who agreed to participate in this study.

2. Gregory A, Kohl BA. Con: near-infrared spectroscopy has not proven its clinical utility as a standard monitor in cardiac surgery. J Cardiothorac Vasc Anesth (2013) 27(2):390-4. doi:10.1053/j.jvca.2012.11.010

3. Hoskote AU, Tume LN, Trieschmann U, Menzel C, Cogo P, Brown KL, et al. A cross-sectional survey of near-infrared spectroscopy use in pediatric cardiac 
ICUs in the United Kingdom, Ireland, Italy, and Germany. Pediatr Crit Care Med (2016) 17(1):36-44. doi:10.1097/PCC.0000000000000564

4. McQuillen PS, Nishimoto MS, Bottrell CL, Fineman LD, Hamrick SE, Glidden DV, et al. Regional and central venous oxygen saturation monitoring following pediatric cardiac surgery: concordance and association with clinical variables. Pediatr Crit Care Med (2007) 8(2):154-60. doi:10.1097/01. PCC.0000257101.37171.BE

5. Chakravarti SB, Mittnacht AJ, Katz JC, Nguyen K, Joashi U, Srivastava S. Multisite near-infrared spectroscopy predicts elevated blood lactate level in children after cardiac surgery. J Cardiothorac Vasc Anesth (2009) 23(5):663-7. doi:10.1053/j.jvca.2009.03.014

6. Daubeney PE, Pilkington SN, Janke E, Charlton GA, Smith DC, Webber SA. Cerebral oxygenation measured by near-infrared spectroscopy: comparison with jugular bulb oximetry. Ann Thorac Surg (1996) 61(3):930-4. doi:10.1016/ 0003-4975(95)01186-2

7. Kussman BD, Wypij D, Laussen PC, Soul JS, Bellinger DC, DiNardo JA, et al. Relationship of intraoperative cerebral oxygen saturation to neurodevelopmental outcome and brain magnetic resonance imaging at 1 year of age in infants undergoing biventricular repair. Circulation (2010) 122(3):245-54. doi:10.1161/CIRCULATIONAHA.109.902338

8. Sood ED, Benzaquen JS, Davies RR, Woodford E, Pizarro C. Predictive value of perioperative near-infrared spectroscopy for neurodevelopmental outcomes after cardiac surgery in infancy. J Thorac Cardiovasc Surg (2013) 145(2):438-45.e1; discussion 44-5. doi:10.1016/j.jtcvs.2012. 10.033

9. Bhalala US, Nishisaki A, McQueen D, Bird GL, Morrison WE, Nadkarni VM, et al. Change in regional (somatic) near-infrared spectroscopy is not a useful indicator of clinically detectable low cardiac output in children after surgery for congenital heart defects. Pediatr Crit Care Med (2012) 13(5):529-34. doi:10.1097/PCC.0b013e3182389531

10. Kurth CD, Steven JL, Montenegro LM, Watzman HM, Gaynor JW, Spray TL, et al. Cerebral oxygen saturation before congenital heart surgery. Ann Thorac Surg (2001) 72(1):187-92. doi:10.1016/S0003-4975(01)02632-7

11. Fenton KN, Freeman K, Glogowski K, Fogg S, Duncan KF. The significance of baseline cerebral oxygen saturation in children undergoing congenital heart surgery. Am J Surg (2005) 190(2):260-3. doi:10.1016/j.amjsurg.2005. 05.023

12. Tume LN, Arnold P. Near-infrared spectroscopy after high-risk congenital heart surgery in the paediatric intensive care unit. Cardiol Young (2015) 25(3):459-67. doi:10.1017/S1047951114000055

13. Kellum JA, Bellomo R, Ronco C. The concept of acute kidney injury and the RIFLE criteria. Contrib Nephrol (2007) 156:10-6. doi:10.1159/000102010

14. Thorniley MS, Livera LN, Wickramasinghe YA, Spencer SA, Rolfe P. The non-invasive monitoring of cerebral tissue oxygenation. Adv Exp Med Biol (1990) 277:323-34. doi:10.1007/978-1-4684-8181-5_38

15. Kussman BD, Laussen PC, Benni PB, McGowan FX Jr, McElhinney DB. Cerebral oxygen saturation in children with congenital heart disease and chronic hypoxemia. Anesth Analg (2017) 125(1):234-40. doi:10.1213/ANE. 0000000000002073

16. Wardle SP, Yoxall CW, Weindling AM. Determinants of cerebral fractional oxygen extraction using near infrared spectroscopy in preterm neonates.
J Cereb Blood Flow Metab (2000) 20(2):272-9. doi:10.1097/00004647200002000-00008

17. Buckley EM, Lynch JM, Goff DA, Schwab PJ, Baker WB, Durduran T, et al. Early postoperative changes in cerebral oxygen metabolism following neonatal cardiac surgery: effects of surgical duration. J Thorac Cardiovasc Surg (2013) 145(1):196.e-205.e. doi:10.1016/j.jtcvs.2012.09.057

18. Greeley WJ, Kern FH, Ungerleider RM, Boyd JL III, Quill T, Smith LR, et al. The effect of hypothermic cardiopulmonary bypass and total circulatory arrest on cerebral metabolism in neonates, infants, and children. J Thorac Cardiovasc Surg (1991) 101(5):783-94.

19. Fleck T, Schubert S, Ewert P, Stiller B, Nagdyman N, Berger F. Propofol effect on cerebral oxygenation in children with congenital heart disease. Pediatr Cardiol (2015) 36(3):543-9. doi:10.1007/s00246-014-1047-7

20. Booth EA, Dukatz C, Sood BG, Wider M. Near-infrared spectroscopy monitoring of cerebral oxygen during assisted ventilation. Surg Neurol Int (2011) 2:65. doi: $10.4103 / 2152-7806.81722$

21. Ricci Z, Haiberger R, Tofani L, Romagnoli S, Favia I, Cogo P. Multisite near infrared spectroscopy during cardiopulmonary bypass in pediatric patients. Artif Organs (2015) 39(7):584-90. doi:10.1111/aor.12424

22. Ghanayem NS, Wernovsky G, Hoffman GM. Near-infrared spectroscopy as a hemodynamic monitor in critical illness. Pediatr Crit Care Med (2011) 12 (4 Suppl):S27-32. doi:10.1097/PCC.0b013e318221173a

23. Daubeney PE, Smith DC, Pilkington SN, Lamb RK, Monro JL, Tsang VT, et al. Cerebral oxygenation during paediatric cardiac surgery: identification of vulnerable periods using near infrared spectroscopy. Eur J Cardiothorac Surg (1998) 13(4):370-7. doi:10.1016/S1010-7940(98)00024-4

24. Brady KM, Mytar JO, Lee JK, Cameron DE, Vricella LA, Thompson WR, et al. Monitoring cerebral blood flow pressure autoregulation in pediatric patients during cardiac surgery. Stroke (2010) 41(9):1957-62. doi:10.1161/ STROKEAHA.109.575167

25. Vretzakis G, Georgopoulou S, Stamoulis K, Stamatiou G, Tsakiridis K, Zarogoulidis P, et al. Cerebral oximetry in cardiac anesthesia. J Thorac Dis (2014) 6(Suppl 1):S60-9. doi:10.3978/j.issn.2072-1439.2013.10.22

26. Pisano A, Galdieri N, Iovino TP, Angelone M, Corcione A. Direct comparison between cerebral oximetry by INVOS(TM) and EQUANOX(TM) during cardiac surgery: a pilot study. Heart Lung Vessel (2014) 6(3):197-203.

Conflict of Interest Statement: This study was investigator initiated but funded by Medtronic including the provision of the Somanetics NIRs device, neonatal, pediatric sensors, and the software necessary to conduct this study. JL is the principal investigator for the grant awarded by Medtronic for the conduct of this study (Project ID: ISR-2014-10423). Medtronic staff was not involved in the study design, execution, and analysis of the results.

Copyright $\odot 2018$ Wong, Chen, Moorakonda, Wijeweera, Tan, Nakao, Allen, Loh and Lee. This is an open-access article distributed under the terms of the Creative Commons Attribution License (CC BY). The use, distribution or reproduction in other forums is permitted, provided the original author(s) and the copyright owner are credited and that the original publication in this journal is cited, in accordance with accepted academic practice. No use, distribution or reproduction is permitted which does not comply with these terms. 